\title{
Elevated Renin Levels Heralds Adrenocortical Involvement In A Case Of Adrenoleukodystrophy
}

\author{
M Beebeejaun, L To, Y Hussain, F Coyle, M Craner, E Bingham and J Tringham
}

\section{Introduction}

Adrenoleukodystrophy (ALD) is an X-linked neurodegenerative disorder of peroxisomal metabolism characterised by the accumulation of very-long-chain fatty acids (VLCFA). ALD has both a neurological and an endocrine interface as VLCFA deposit in the central and peripheral nervous system as well as the adrenal cortex and testis. Clinical presentation is therefore widely heterogeneous. ALD can present as adrenocortical failure with or without neurological complications. It is imperative that all patients diagnosed with ALD have adrenal reserve assessments. Elevated renin levels is a subtle indication that should prompt regular investigations to prevent an Addisonian crisis during the course of the disease.

\section{Case presentation}

We describe the case of a 40 year old gentleman who presented with progressive spastic paraparesis following a road traffic accident. He was fit and well and has 2 children with no previous fertility issues. Neurological imaging did not reveal any structural or demyelinating abnormalities. After extensive investigations, he was noted to have elevated levels of VLCFA and genetic tests confirmed a mutation in the $A B C D 1$ gene; hence confirming a diagnosis of ALD ( See Fig1 ). An endocrine work-up was carried out. He described fatigue and minimal postural symptoms. He had also experienced erectile dysfunction since the accident. On examination, he was not obviously hyperpigmented and demonstrated no postural blood pressure changes. Further tests revealed normal sodium and potassium levels, a normal aldosterone level of $180 \mathrm{pmol} / \mathrm{L}(100-800)$ and an elevated renin level of $78.8 \mathrm{mu} / \mathrm{L}(12.9-33.7)$. His 9am serum testosterone level was on the low side of the normal range with a high FSH and normal LH level, indicating spermatogenic failure (see Table 1). A short synacthen test showed a normal cortisol response to ACTH with a 30 minute cortisol of $603 \mathrm{mmol} / \mathrm{L}$. (See Table 2) He was started on Fludrocortisone and has 6 monthly adrenal reserve measurements.

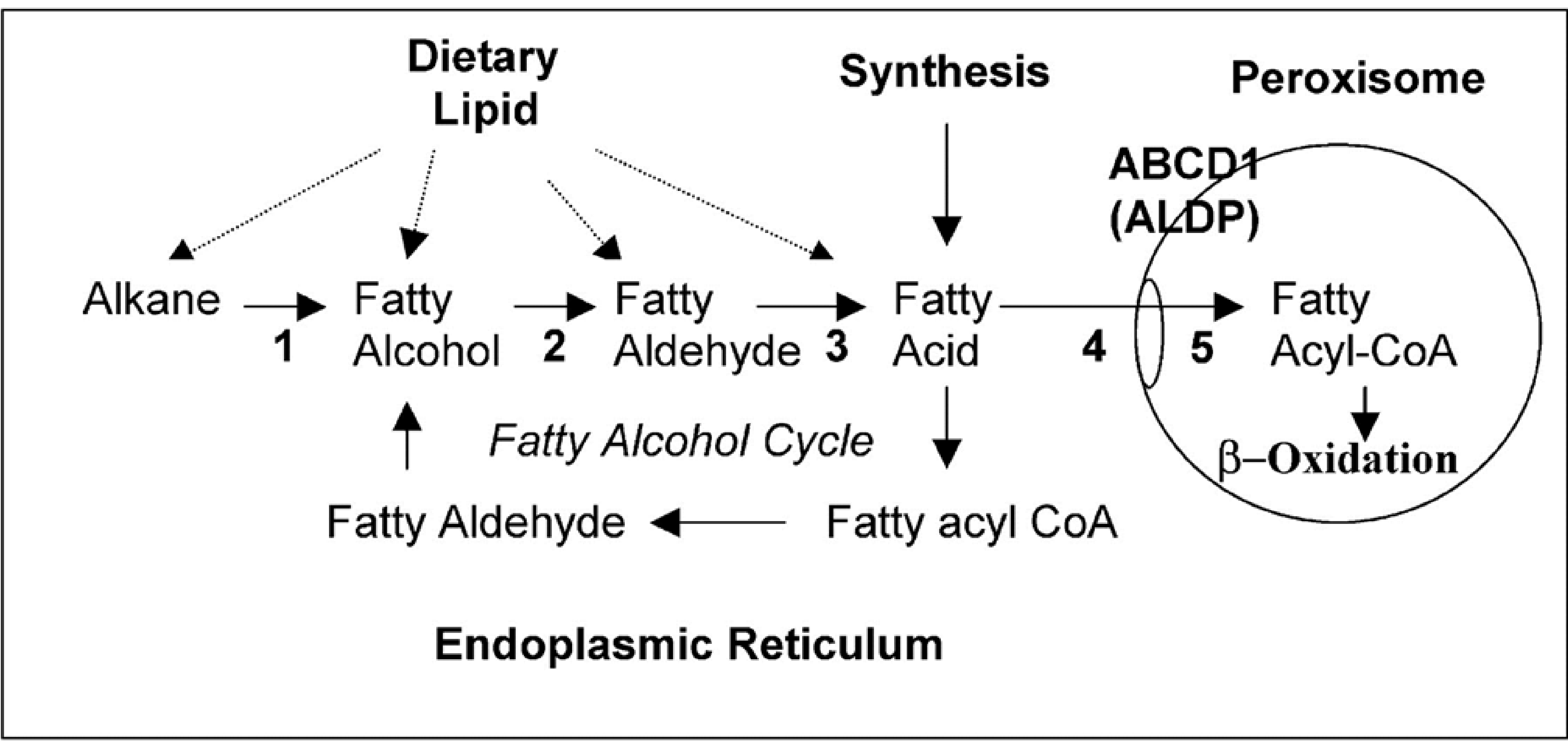

Figure 2. Pathway of fatty acid catabolism, peroxisomal oxidation and the role of the ABCD1 gene

\section{Discussion}

X-linked ALD is characterised biochemically by impaired beta oxidation in peroxisomes which results from a mutation in the ABCD1 gene. ( See Fig 2 and Fig 3) ALD can present in childhood with a cerebral form or later in life as an adrenomyeloneuropathy type. There is no clinical correlation between the severity of the neurological presentation and the endocrine manifestations. Adrenal insufficiency may coexist with the neurological dysfunction or develop during the progression of the disease as more VLCFA deposit in adrenal tissues. A short synacthen test will reliably evaluate the cortisol reserve. However, it is important to identify those patients who would be more at risk of developing an adrenal crisis. Serial plasma ACTH have been used in some cases for this purpose. An elevated renin level is also an indication of subclinical adrenal involvement as this indicates deposition of VLCFA in the zona glomerulosa ; hence predicting those patients with ALD who need more frequent monitoring. Our case presented with neurological sequelae but has a normal cortisol response to ACTH. There was also no overt mineralocorticoid deficiency as the aldosterone levels were within the normal range. A high renin level in this patient is reflecting early adrenal VLCFA deposition. Screening this patient for adrenal insufficiency in the future would be therefore more rigorous.

\section{ABCD1 Sequencing Result of patient \\ Hemizygous for c.1252>T, p.( Arg418Trp)}

\section{Test Methodology}

Fluorescent sequence analysis of exons 1 to 10 of the $A B C D 1$ gene. Whole exon deletions and duplications of the ABCD1 gene using the P649-C1 MLPA ( Multiplex ligation-dependent probe amplification) kit from MRC-Holland

\section{Results}

Misense mutation, c.1252>T, p. ( Arg418Trp) in exon 4 of the ABCD1 gene. This patient has a pathogenic mutation reported in families with Adrenoleukodystrophy

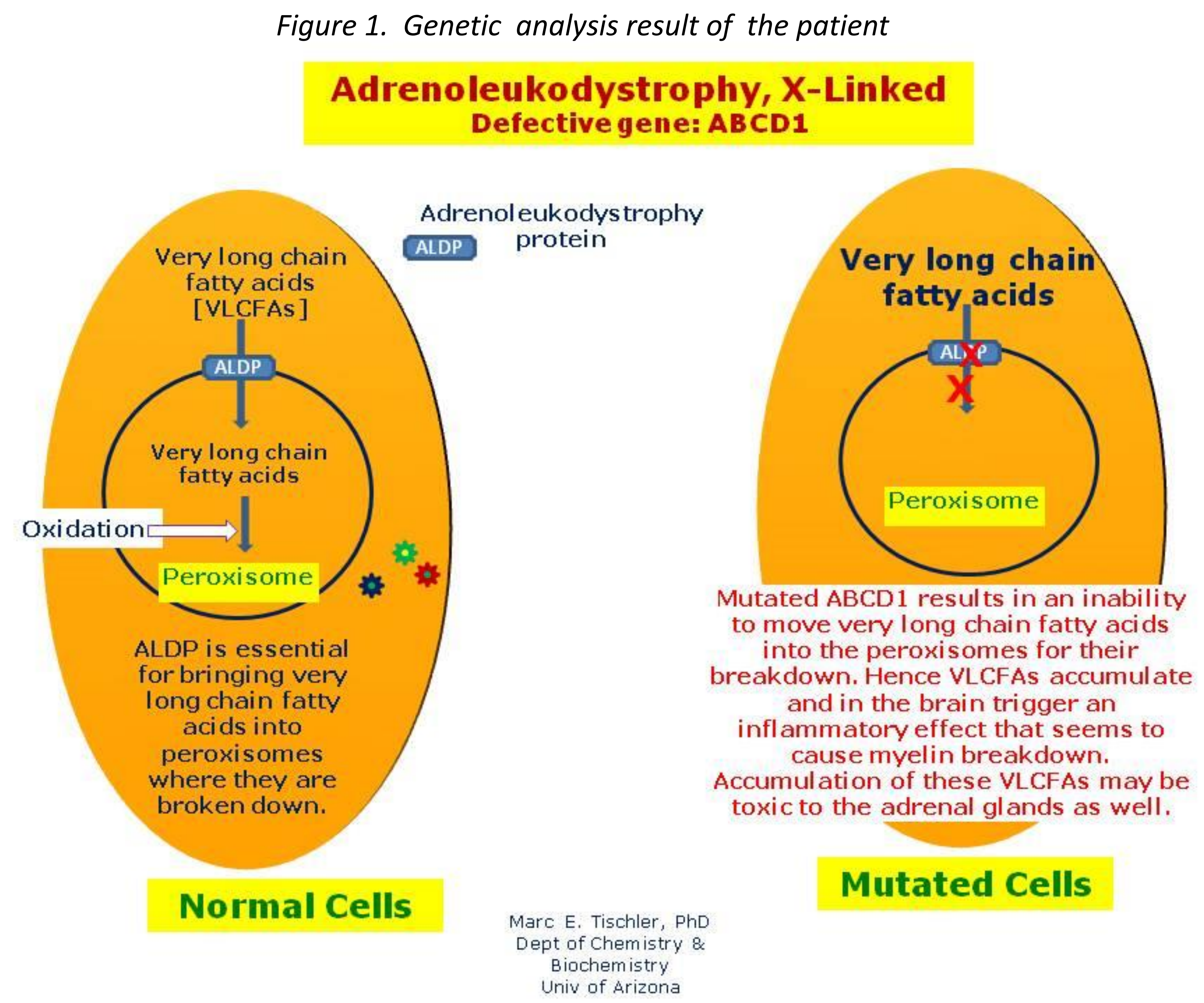

Figure 3. Illustration of cell mutation with a defective $A B C D 1$ gene

\begin{tabular}{ll}
\hline Na & $\mathbf{1 4 0 ~ m m o l} / \mathrm{L}$ \\
\hline K & $4.0 \mathrm{mmol} / \mathrm{L}$ \\
Urea & $5.9 \mathrm{mmol} / \mathrm{L}$ \\
Creat & $74 \mu \mathrm{mol} / \mathrm{L}$ \\
eGFR & $>60 \mathrm{ml} / \mathrm{min}$ \\
\hline Aldosterone & $180 \mathrm{pmol} / \mathrm{L}(100-800)$ \\
\hline Renin & $78.8 \mathrm{mu} / \mathrm{L}(12.9-33.7)$ \\
& \\
\hline 9am cortisol & $510 \mathrm{nmol} / \mathrm{L}$ \\
\hline Serum testosterone & $10.4 \mathrm{nmol} / \mathrm{L}$ \\
\hline SHBG & $52.9 \mathrm{nmol} / \mathrm{L}$ \\
\hline Free testosterone & $0.146 \mathrm{nmol} / \mathrm{L}$ \\
LH & $6.4 \mathrm{IU} / \mathrm{L}$ \\
FSH & $23.1 \mathrm{IU} / \mathrm{L}$ \\
\hline
\end{tabular}

Table 1 Blood test Results

\section{Time/min Cortisol/ $\mathrm{nmol} / \mathrm{L}$}

\begin{tabular}{|c|c|}
\hline 0 & 365 \\
\hline 30 & 603 \\
\hline 60 & 610 \\
\hline
\end{tabular}

Table 2 Short Synacthen test

\section{Conclusion}

This case highlights the importance of carefully screening all ALD cases for adrenocortical involvement; this will prevent catastrophic Addisonian crises in periods of stress. Elevated renin levels can herald impending adrenal cortex failure further during the course of the disease; hence the need for regular adrenal reserve assessments. Likewise, male patients diagnosed with idiopathic or negative adrenal antibody Addison's disease should_have VLCFA measured to exclude_ALD. 